\title{
La théorie du macrosystème interlectal et l'acquisition langagière en contexte plurilingue : réflexion sur la situation réunionnaise
}

\author{
Noël, Audrey \\ Université de La Réunion. LCF, EA 4549 \\ naudrey86@aol.com
}

\section{Introduction}

La psycholinguistique se pose de nombreuses questions concernant la manière dont la modalité langagière est acquise, lorsqu'un enfant maîtrise deux ou plusieurs codes linguistiques. Les recherches dans le domaine de l'acquisition se donnent alors pour but d'éclairer le débat théorique concernant le fonctionnement et l'organisation cognitive des individus plurilingues: les locuteurs bilingues ou plurilingues possèdent-ils un système linguistique unique, ou duel ? Y-a-t-il, au cours du développement langagier, changement de fonctionnement linguistique, ou celui-ci est-il identique tout au long de la vie ?

Nous nous intéresserons dans cet article à l'acquisition langagière des individus plurilingues, en prenant pour objet d'étude La Réunion. Ce département français de l'océan Indien présente une situation toute particulière de bilinguisme : la cohabitation de deux codes linguistiques (français et créole réunionnais) donne à entendre des phénomènes abondamment décrits par la littérature (code-switching, emprunt, calque...), mais aboutit également à la production fréquente d'énoncés mélangés, non clairement rattachables à l'une ou à l'autre langue. L'originalité de ces pratiques linguistiques, attestées dans d'autres aires créolophones (Martinique par exemple), amène à envisager que les langues des locuteurs réunionnais fonctionneraient à un degré maximal d'interrelation, se fondant en un système linguistique unique, un macrosystème de communication (Prudent, 1981 ; Souprayen-Cavery, 2010).

Cette façon innovante de concevoir l'organisation linguistique de locuteurs plurilingues n'est pas sans amener un certain nombre d'interrogations et nourrit la réflexion théorique sur l'acquisition : en effet, dans un tel cadre conceptuel, comment s'organiserait l'acquisition langagière ? Peut-on toujours raisonner en termes de langue maternelle, de langue seconde? Quelles sont les implications théoriques, épistémologiques, concernant l'organisation linguistique sur le plan cognitif?

Nous proposons d'effectuer tout d'abord un survol théorique concernant l'acquisition langagière de l'enfant bilingue et les pratiques linguistiques effectives des jeunes enfants réunionnais, ce qui nous amènera à développer le concept du macrosystème interlectal. Nous verrons ensuite en quoi ce cadre théorique remet en question, voire déconstruit, certaines notions classiquement usitées pour parler des situations bilingues. Si effectivement certains repères théoriques semblent devenir inopérants au vu de l'originalité de la situation linguistique réunionnaise, d'autres peuvent être avancés pour mieux comprendre la manière dont l'enfant réunionnais acquiert le langage et s'érige en être plurilingue ; c'est ce que nous aborderons dans un troisième temps, avant de clore notre propos. 


\section{Données théoriques}

\subsection{L'acquisition langagière de l'enfant bilingue}

\subsubsection{Trajectoire, rythme et facteurs de variation}

La littérature a cherché à savoir en quoi le développement langagier des enfants bilingues ou plurilingues se distingue du développement des enfants monolingues. Il ressort aujourd'hui des différentes recherches que le développement langagier des bilingues suit la même trajectoire (étapes, âges d'acquisition) que celui des monolingues (Hamers, 1997 ; Paradis et al., 2005b ; Rivera Mindt et al., 2008), mais présente cependant quelques particularités. Ainsi le rythme des acquisitions peut être modifié (accélération ou décélération; Paradis et Genesee, in Paradis et al., 2003), et l'on peut également observer des phénomènes de transfert (une structure linguistique peut par exemple être utilisée dans l'autre langue, et ce pour une période temporaire du développement). Dans tous les cas, les caractéristiques structurelles des langues de l'enfant, et leurs possibles points communs (cas des langues génétiquement proches) vont influencer le développement langagier. L'élaboration des compétences linguistiques des enfants bilingues est également soumise à de nombreux facteurs de variation, qui accentuent les différences entre les individus bilingues (Paradis, 2007a). Ont par exemple une influence :

la motivation à acquérir les langues, elle-même influencée par les milieux familial et social, et par le statut sociolinguistique des langues (langue officielle / langue minoritaire) ;

les aptitudes et la personnalité de l'individu ;

l'âge d'acquisition des langues ;

la qualité et les contextes d'input linguistique.

\subsubsection{Conséquences cognitives du bilinguisme selon les recherches en psycholinguistique}

Certaines recherches se sont quant à elle intéressées aux avantages du bilinguisme (Hamers, 1997 ; Köpke, 2007 ; Rivera Mindt et al., 2008). Il a été mis en évidence que lorsque certaines conditions favorables sont réunies, l'enfant développe non seulement de bonnes compétences linguistiques dans chacune de ses langues, mais tire également bénéfice, sur le plan cognitif, de sa double inscription langagière : les sujets bilingues auraient en effet de meilleures capacités d'inhibition et de contrôle, ce qui impacterait de façon positive sur les fonctions exécutives (les processus cognitifs permettant un comportement flexible et adapté au contexte : capacités d'anticipation, de planification...). D'autres auteurs sont plus nuancés (Alario, 2012): si être bilingue confère très certainement de nombreux avantages, il existerait également certains inconvénients, par exemple une plus grande lenteur d'accès au lexique, de sélection et d'articulation de l'item cible. Si les sujets bilingues développent une plus grande habilité à utiliser le mécanisme d'inhibition, ce dernier, constamment à l'œuvre, traduit que le passage de l'une à l'autre langue demande un effort non négligeable.

L'organisation cognitive des langues est également un sujet longuement abordé par la psycholinguistique (Hamers et Blanc, 1983) : se pose ainsi la question de l'unité, ou au contraire de la séparation, des différentes langues de l'individu bilingue ou plurilingue. Les théories divergent en fonction des composantes langagières étudiées. En ce qui concerne la composante phonologique, plusieurs théories sont aujourd'hui avancées (Goldstein et Fabiano, 2007) :

la théorie du Système Unitaire (Unitary System Model ; Paradis, 2001 ; Bhatia et Ritchie, 1999), qui postule au début de l'acquisition langagière un système phonologique unique, se séparant en deux systèmes autonomes au fil du temps ; 
la théorie du système duel (Dual Systems Model; Keshavarz et Ingram, 2002), qui postule au contraire que l'enfant bilingue posséderait dès la naissance deux systèmes distincts, qui le resteraient tout au long de la vie ;

et le système interactionnel (Interactional Dual Systems Model; Paradis, 2001), qui postule que l'enfant bilingue posséderait deux systèmes phonologiques en constante interaction. L'enfant utiliserait donc ses deux systèmes phonologiques afin d'assurer l'efficience de sa production, et différencierait ses systèmes lorsque cela est requis.

Pour Alario (2012), l'organisation du système phonologique dépendrait en grande partie du type de bilinguisme : certaines tâches expérimentales montrent en effet que les locuteurs bilingues précoces (acquisition de deux langues avant 4 ans) possèdent des compétences phonologiques et une prononciation proches de celles de locuteurs monolingues, ce qui suggère que les représentations sont tenues séparées ; au contraire, les bilingues tardifs (acquisition après l'âge de 6 ans) gardent fréquemment un accent, ce qui appuie l'hypothèse que les représentations sont communes. L'unité des systèmes linguistiques concernerait donc les sujets bilingues tardifs et provoquerait des imperfections linguistiques.

Pour ce qui est de la composante lexicale, la question majeure reste la même : les théories vont de l'hypothèse d'une représentation coordonnée (Weinreich, 1953), ce qui sous-entend deux lexiques distincts en interdépendance, à l'hypothèse d'une représentation composée (un seul système lexical pour les deux langues). Les approches les plus récentes concilient les deux points de vue : il y aurait à la fois deux lexiques distincts, et une base conceptuelle commune, aboutissant à une représentation mixte, à la fois composée et coordonnée (Kroll et Stewart, in Lavaur, 1996).

La question est moins abordée dans les travaux portant sur la morphosyntaxe, qui s'avère être la composante la plus complexe à analyser. En effet, le développement morphosyntaxique est fortement dépendant des langues parlées par l'enfant, de leur organisation structurelle spécifique, et de leur degré de similitude (Mackey, 1997). Il y a influence de la complexité des règles grammaticales sur l'acquisition (Lindholm, 1980): le développement morphosyntaxique de l'enfant bilingue ne peut donc être appréhendé que par l'analyse des systèmes linguistiques spécifiques des langues. Il s'avère difficile en ce cas de postuler des représentations morphosyntaxiques uniques ou distinctes tant cela semble lié à la nature des langues parlées par l'individu bilingue.

\subsubsection{Plurilinguisme et pratiques hybrides attestées}

Si les enfants bilingues apprennent au cours de leur développement langagier plusieurs langues, ils vont de fait également les mélanger : ce phénomène est propre aux comportements langagiers bilingues (Hamers et Blanc, 1983; Paradis et al., 2005a; Goldberg et al., 2008; Abdelilah-Bauer, 2008). De nombreuses familles plurilingues fonctionnent sur un «mode bilingue » (elles conservent l'usage, dans la cellule familiale, des deux langues), les usages étant plus ou moins libres et aboutissant à des productions mélangeantes (Deprez, 1989). Les pratiques linguistiques familiales sont également largement influencées par la situation socio-culturelle de la famille et par les usages de la communauté linguistique dans laquelle elle évolue (famille immigrée dans une communauté linguistique monolingue versus famille évoluant dans sa propre communauté plurilingue ; Deprez, 2002 ; Rapanoël et Ledegen, 2007 ; Marvilliers, 2009). C'est à partir de l'input linguistique (de la famille et/ou du groupe social) et des feedback correctifs que l'enfant acquiert le matériau langagier et peut développer la capacité à mélanger.

Plusieurs formes de mélange sont décrites par la littérature : on parle principalement de code-switching, ou alternance des codes, d'emprunt, et de calque (Hamers et Blanc, 1983 ; Hamers, 1997; Lefebvre, 2008). L'alternance des codes désigne la juxtaposition, à l'intérieur d'un même échange verbal, d'énoncés appartenant à deux langues différentes. Celle-ci ne se fait pas au hasard : elle est sous-tendue par des règles, que l'enfant apprend très tôt grâce à l'input linguistique reçu. L'emprunt désigne quant à lui l'utilisation d'un morphème ou d'une expression d'une langue, intégré(s) dans une autre sans traduction. Il s'agit d'un phénomène restreint au domaine lexical, et généralement considéré comme conscientisé par le locuteur. Le calque est une forme particulière d'emprunt, puisqu'il concerne un syntagme entier qui est 
transposé d'une langue à une autre, et traduit de façon littérale (par exemple, « fall in love » qui a abouti à l'expression « tomber en amour » en québécois).

On trouve encore chez certains auteurs la description d'une autre forme de mélange, l'interférence. L'interférence est un phénomène jugé inconscient, pouvant se manifester à tous les niveaux de production linguistique (phonémique, lexical, syntaxique...). Contrairement aux autres phénomènes décrits, le terme « interférence » induit un jugement de valeur (Tirvassen, 2003) : en effet, l'interférence est considérée comme une déviance, une intrusion inappropriée d'un code dans un autre (Hamers et Blanc, 1983), qui serait principalement retrouvée chez des locuteurs ayant une connaissance limitée de la langue qu'ils utilisent. Dans le domaine pédagogique, elle est synonyme d'erreur. La définition de l'interférence illustre bien le fait que le mélange est encore bien souvent considéré avec méfiance : si les sociolinguistes estiment ce phénomène normal et typique des situations plurilingues, d'autres linguistes continuent de penser qu'il peut être signe de difficulté, de confusion linguistique. Pour Abdelilah-Bauer (2008), cette image de la confusion se nourrit de l'idée scientifique du système linguistique unique, qui serait celui des jeunes enfants n'ayant pas encore conscientisé qu'ils possèdent plusieurs codes linguistiques, et des bilingues tardifs; ceci se traduirait par des différences plus prégnantes par rapport au groupe des monolingues (accent plus marqué, accès au lexique plus lent...). Or le mélange se retrouve chez tout type de locuteur bilingue, s'atteste dans toute conversation où les participants sont plurilingues, et peut même, pour certains auteurs, être le signe d'un haut niveau de compétence linguistique (Borel, 2004 ; Coste et al., 1999). Que pouvons-nous dire alors des pratiques linguistiques des locuteurs de La Réunion, île caractérisée par une situation de contact de langues, et plus particulièrement des pratiques linguistiques des jeunes enfants réunionnais ? Atteste-t-on également du code-switching, de l'emprunt, des calques?

\subsection{Les pratiques langagières à La Réunion}

\subsubsection{Présentation de La Réunion}

L'île de La Réunion, département français depuis 1946, est une terre émergée d'une superficie de 2512 $\mathrm{km}^{2}$, située dans l'océan Indien à 700 kilomètres à l'est de Madagascar. Le dernier recensement INSEE de 2012 estime la population réunionnaise à 837868 habitants. Celle-ci est plurielle : plusieurs groupes ethniques sont représentés (communautés chinoise, hindoue, malgache, mahoraise, comorienne...), et se sont mélangés au fil du temps ; une grande partie de la population réunionnaise est donc métissée, et si les différents groupes ethniques conservent leurs caractéristiques identitaires et culturelles, tous partagent un sentiment d'appartenance à la communauté réunionnaise. Ce sentiment repose en grande partie sur le facteur linguistique : le créole réunionnais, créole à base française né à la période de l'esclavage, est parlé par une grande majorité de la population. La moitié des Réunionnais de 16-64 ans se considèrent par ailleurs unilingues créoles, tandis que 38\% des Réunionnais se disent bilingues (Monteil, 2010). Le français est la langue officielle de l'île ; sont également parlées des langues minoritaires (langue tamoule, malgache, shi maore...), dont l'usage est encore vivace chez les familles récemment immigrées (cas par exemple du shi maore).

\subsubsection{Pratiques linguistiques des Réunionnais : description et théorisation}

Les Réunionnais «affirment parler "créole » ou «français », [mais] nous entendons au-delà de ces premières catégories une «parole mélangée » (Souprayen-Cavery, $2010: 16$ ). Illustrons cette citation par deux extraits de corpus de locuteurs adultes, recueillis sur une chaîne de radio locale :

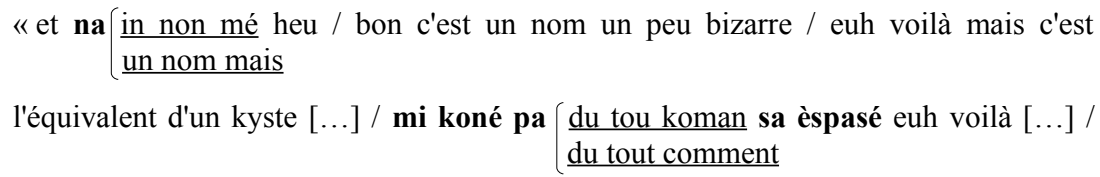




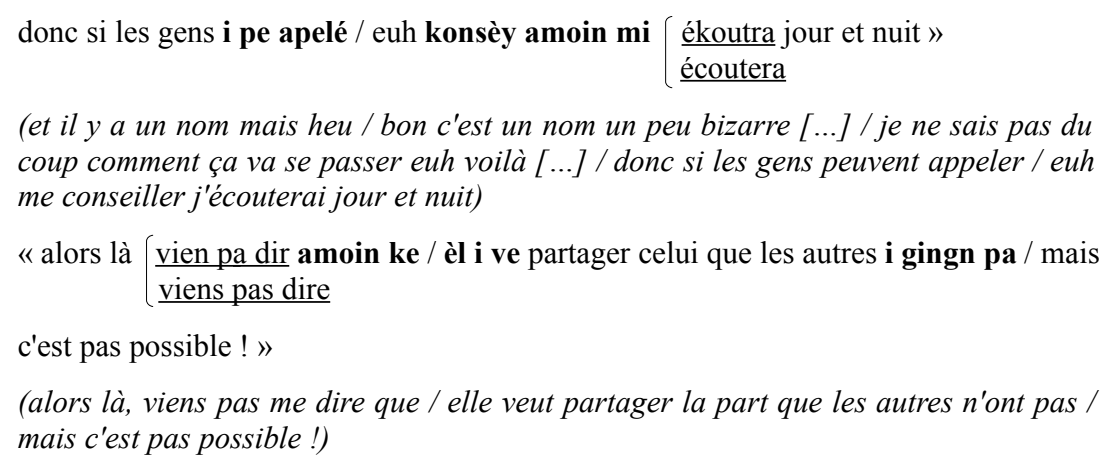

On atteste ici des segments en français (police simple dans les extraits), des segments en créole, transcrits en graphie phonologisante, contenant des marqueurs prototypiques créoles décrits par les grammaires (en gras), et également des segments mixtes (soulignés dans les extraits), qui peuvent être attestés en créole comme en français. Pour matérialiser ce fait, nous avons choisi de proposer une double transcription de ces segments, en nous inspirant de la méthodologie proposée par Ledegen (2012) ; cette manière de procéder permet de ne pas prendre parti pour un des codes linguistiques et inscrit la démarche du linguiste dans une approche interlectale. Signalons néanmoins que dans certains cas, les phénomènes prosodiques permettent au linguiste de statuer sur la langue ciblée par le locuteur.

Ces deux extraits montrent que les locuteurs réunionnais alternent les codes linguistiques à haute fréquence : on retrouve donc les phénomènes de mélange décrits par la littérature, mais leur proportion amène à envisager l'ensemble des énoncés comme étant métissés. D'autres énoncés font apparaitre des formes plus inattendues, comme dans l'extrait ci-dessous :

« ouais / voilà / bon ben bon appétit à tout le monde sèt $\mathrm{i}$ von passer à table » (ouais / voilà / bon ben bon appétit à ceux qui vont passer à table)

Dans cet extrait, «sèt $i$ von » est un segment qui n'est prédictible ni par la grammaire du créole (on attendrait par exemple « sèt i sa », ou encore « sat i sava »), ni par celle du français. Il n'est pourtant pas $a$ priori jugé inacceptable, si l'on tient compte de la réaction neutre de l'interlocuteur lors de l'échange. Il s'agit néanmoins d'une forme agrammaticale, car échappant à la grammaire.

Ces segments ambigus ou hors grammaire témoignent de la forte osmocité entre le français et le créole (Chaudenson, 1993). Par ailleurs, l'absence de cadre normatif consensuel pour le créole (malgré l'existence de descriptions grammaticales) rend la délimitation d'autant plus difficile. La parole réunionnaise semble alors

«se construire entre le créole et le français sans qu'il soit possible de la catégoriser
formellement selon une appartenance au créole acrolectal ou basilectal, au français
créolisé, au français régional et au créole francisé » (Souprayen-Cavery, 2010:21).

Dans quel courant théorique se placer pour décrire cette situation linguistique plutôt atypique ? Prudent (1981) fait le constat, à la suite de ses travaux sur la situation linguistique martiniquaise, que l'on observe dans les productions langagières des locuteurs martiniquais de l'alternance codique, phénomène tout à fait caractéristique des situations de contact de langues, dont l'analyse détaillée peut permettre de comprendre les contextes d'apparition, mais présent à si forte fréquence qu'une telle analyse serait fastidieuse pour le linguiste. Il note que l'on observe aussi la présence de segments non prévisibles et non explicables. La rencontre de deux grammaires produirait en effet des formes originales, qui ne relèvent clairement du «moule » d'aucune des deux langues, et qui peuvent survenir dans tous les contextes sociolinguistiques. Ceci l'amène alors à proposer le concept de l'interlecte, ou macrosystème interlectal. Ce modèle théorique 
fait par la suite son apparition à La Réunion dans les années 90 (Ledegen, 2003 ; Georger, 2005 ; Souprayen-Cavery, 2010; Ledegen, 2012). Ainsi, il existerait, en sus du créole et du français, une

«zone interlectale se présent[ant] [...] comme l'ensemble des paroles qui ne peuvent être prédites par une grammaire de l'acrolecte ou du basilecte» (Prudent, 1981).

Cette zone interlectale serait partie intégrante d'un macro-système comprenant toute une gamme de variations engendrées par le contact des langues (Lebon-Eyquem, 2010a). Le macrosystème serait alors composé de plusieurs lectes, ou sous-systèmes : le français avec toute sa variation, le créole avec toute sa variation, et des formes interlectales, prévisibles ou non prévisibles. Le macrosystème de communication recouvre toutes les formes langagières s'étendant entre deux pôles extrêmes, le français standard d'une part et le créole basilectal de l'autre, considéré comme la forme linguistique la plus différente du français. Le locuteur réunionnais posséderait donc une macrolangue. Simonin (2002) décrit quant à lui l'interlecte comme la manifestation d'une phase d'hybridation des parlers, qui rend compte de la dynamique sociolinguistique des langues en contact : il propose le terme de « réunionnais » pour qualifier la langue parlée par les locuteurs de La Réunion, de la même manière que l'on nomme « français » la langue parlée en France, en dépit de ses nombreuses variétés.

D'autres concepts avaient été précédemment élaborés pour rendre compte des pratiques linguistiques des locuteurs réunionnais, comme celui du continuum linguistique, reliant les différentes variantes linguistiques en interrelation (Beniamino et Baggioni, 1993). Le concept du macrosystème interlectal s'avère novateur et plus opérant, car il rend non seulement compte des pratiques linguistiques panachées des Réunionnais, mais amène également à réhabiliter le phénomène du mélange. Voyons maintenant ce qu'il en est des pratiques linguistiques des jeunes enfants réunionnais.

\subsubsection{Les pratiques linguistiques des jeunes enfants réunionnais}

Le jeune enfant réunionnais évolue dans un environnement où il est en contact avec plusieurs langues, mais également en contact avec une parole mélangée ; comment se caractérisent alors ses productions linguistiques? Sont-elles comparables à celles des locuteurs adultes, ou présentent-elles au contraire certaines particularités ? L'enquête menée par Fioux (2007) auprès d'enfants scolarisés en grande section de maternelle a montré que les langues sont effectivement souvent mélangées. Le langage des jeunes enfants réunionnais reste typique de celui des enfants de 4-5 ans : on observe, en sus des productions mélangées, des approximations langagières sur les plans phonologique et syntaxique. Lebon-Eyquem (2010b) a quant à elle proposé une typologie des énoncés produits par les enfants réunionnais; on peut donc distinguer :

des formes du français (« il a déjà couru »);

des formes créoles contenant des morphèmes prototypiques de cette langue («li la boir in pe delo »: « il a bu un peu d'eau »), matérialisées en gras dans les exemples ;

des essais intrasystémiques : l'enfant émet des hypothèses tâtonnantes en se basant sur un seul code, à la manière d'un enfant monolingue (« il a coudé » pour « il a cousu »);

et des formes mélangeantes, métissées, comprenant deux grands types de formes linguistiques, présentées dans le tableau ci-dessous : 


\begin{tabular}{|l|c|}
\hline \multicolumn{3}{|c|}{$\begin{array}{c}\text { Formes métissées socialement établies } \\
\text { (acceptables par la communauté linguistique) }\end{array}$} \\
$\begin{array}{l}\rightarrow \text { alternances codiques intra-énoncés (segments } \\
\text { créoles accolés à des segments français) ou inter- } \\
\text { énoncés (énoncés créoles accolés à des énoncés } \\
\text { français) }\end{array}$ & " ma la parti la kaz akoz / euh il y avait pas \\
d'école »
\end{tabular}

· Tableau 1. Typologie des formes métissées, ou interlectales (Lebon-Eyquem, 2010b)

Cette catégorisation des pratiques, réalisée à partir d'analyses linguistiques de corpus d'interactions spontanées, met en évidence la grande diversité des pratiques linguistiques des jeunes enfants réunionnais. Ces derniers, tout comme les locuteurs adultes réunionnais, ne se contentent pas de parler français ou créole : ils produisent des énoncés métissés, tantôt acceptables (d'après le jugement linguistique de l'interlocuteur), tantôt non acceptables. La capacité à mélanger requiert donc bien un apprentissage, et son acquisition se traduit par la production d'énoncés tâtonnants qui sont provisoires. Le mélange produit par l'enfant réunionnais n'est donc pas seulement une étape provisoire du développement langagier, une «confusion » temporaire des codes, car non seulement il ne disparaît pas à l'âge adulte, mais il va au contraire se pérenniser et s'affirmer.

Le macrosystème interlectal amène donc un nouvel éclairage sur les productions langagières, mais amène également de nouvelles interrogations dans une perspective acquisitionniste : l'enfant réunionnais, évoluant dans une situation linguistique particulière, doit s'approprier des lectes qui sont étroitement imbriqués et forment un tout macro-systémique. Comment concevoir les étapes de l'acquisition langagière dans ce cadre théorique ? Les notions issues des études sur le bilinguisme peuvent-elles s'appliquer en contexte réunionnais ? Il apparaît en effet que ce nouveau modèle à penser le langage des Réunionnais nous amène à remettre en question un certain nombre de concepts.

\section{Déconstruction des concepts envisageant l'acquisition langagière comme un processus successif}

\subsection{Langue maternelle (L1), langue seconde}

Il est encore d'usage, face à un individu bilingue, de chercher à définir sa langue maternelle. Dans le cas du bilinguisme consécutif (acquisition après 4 ans), celle-ci semble facilement identifiable : la langue maternelle aura donc le statut de langue première (ou L1), tandis que la langue secondairement acquise aura le statut de L2. Dans les cas de bilinguisme précoce, les notions de langue maternelle et de langue seconde s'avèrent moins pertinentes. Qu'entend-on véritablement par « langue maternelle »? Force est de constater que le terme souffre déjà d'une imprécision théorique. 
Moore (2006) rappelle que le terme « langue maternelle» désigne en tout premier lieu la langue de la mère, la langue du foyer. Mais la définition de la langue maternelle est en réalité plurielle et repose sur cinq critères majeurs :

- le lien avec la langue parlée par la mère ;

- l'antériorité de l'appropriation d'une des langues ;

- le niveau de compétence atteint ;

- le concept de mode naturel d'acquisition (qui s'oppose à l'idée de l'apprentissage) ;

et enfin l'organisation ou le fonctionnement de la fonction langagière.

Il apparaît donc hasardeux de vouloir identifier la « langue maternelle » d'un individu dans un contexte de plurilinguisme, car derrière ce terme peut se cacher une réalité très différente selon le critère définitoire adopté. Abdelilah-Bauer (2008) souligne également à quel point l'identification chez un enfant bilingue de la langue maternelle, de la langue première, peut être problématique : quelle serait la langue maternelle d'un enfant qui évolue depuis son plus jeune âge dans un environnement où deux langues sont utilisées quotidiennement? Pour cette auteure, la langue maternelle des enfants bilingues n'est autre que le bilinguisme lui-même, tant il est parfois difficile de différencier les langues et de leur attribuer un ordre d'acquisition.

À La Réunion, la question de la langue maternelle se pose de façon plus prégnante : l'enfant réunionnais évolue dans un univers plurilingue, et il est confronté à une parole mouvante, à un mélange à géométrie variable, car celui-ci ne peut être clairement rattaché ni à un individu particulier, ni à une situation précise. Tout un chacun peut mélanger... Ou ne pas mélanger. Il peut être tentant, au premier abord, de penser que la langue maternelle de l'enfant réunionnais est forcément la langue créole, à partir du moment où il évolue dans un milieu créolophone. Or dire ceci revient à postuler qu'un enfant créolophone n'entendrait pas parler français autour de lui durant sa petite enfance, ou trop peu pour que cette langue puisse être un moyen d'expression linguistique, ce qui ne reflète pas la réalité quotidienne : le français est au minimum présent dans le foyer familial par le biais des médias et des nouvelles technologies (télévision, radio, ordinateur, jeux électroniques...), et l'on sait les foyers réunionnais relativement bien équipés à ce niveau.

De la même manière, il est peu plausible qu'un enfant réunionnais n'ait pas été en contact avec le créole durant sa petite enfance: dans l'éventualité où le créole serait proscrit dans le milieu familial, et le français privilégié (il s'agit ici de politique linguistique familiale ; Crémieux, 2000 ; Noël, 2012), les parents déclarent souvent que le créole leur « échappe » dans des contextes émotionnels forts, ou continue à être parlé entre les adultes. De plus, le créole n'est plus désormais restreint à un usage oral et intime : il s'entend et s'écrit dans les journaux, à l'école, sur les panneaux publicitaires, sur les chaînes locales.

Il se peut donc dans certains cas que la langue maternelle de l'enfant réunionnais ne soit ni le français, ni le créole, mais les deux, autrement dit le "réunionnais ». C'est à cette même conclusion que sont parvenus Genelot et al. (2006) pour la situation martiniquaise : l'environnement sociolinguistique de l'enfant martiniquais semblerait mettre en présence deux langues dès le plus jeune âge, langues qui se développent simultanément mais à des rythmes différents en fonction des pratiques langagières familiales. S'il ne s'avère pas pertinent de parler de langue maternelle (ou L1) et de L2, on peut alors s'interroger sur l'adéquation de certaines théories développées à partir de ce cadre à penser la(les) langue(s).

\subsection{Cummins et la théorie des seuils}

Cummins (1976), s'appuyant sur les travaux de Lambert, postule qu'un enfant bilingue doit atteindre un premier seuil de compétence dans sa L1, pour éviter l'apparition d'un handicap linguistique (bilinguisme soustractif), et un deuxième seuil de compétence, en L1 et en L2, pour que la situation de bilinguisme soit harmonieuse et lui permette de tirer profit, sur le plan cognitif, de sa double compétence linguistique. Il 
est donc indispensable d'introduire la L2 lorsque la L1 est déjà bien acquise, et de conserver l'usage de la L1 par la suite.

La théorie des seuils présente l'avantage certain de militer en faveur du maintien de la L1 durant l'enfance et la scolarisation des enfants bilingues, agitant le spectre du handicap linguistique ; Cummins a été à bon escient largement cité, à La Réunion, pour alerter sur l'absence de la prise en compte de la langue créole durant la scolarisation. Nous pouvons néanmoins nous interroger sur son adéquation théorique à la situation plurilingue de façon générale, et plus spécifiquement à la situation linguistique réunionnaise. En effet, s'il n'y a pas lieu de parler de L1 et de L2, comment concevoir qu'il puisse exister des seuils de compétences linguistiques à atteindre dans chaque langue ?

À La Réunion, le problème est plus complexe : il existe une véritable problématique de l'échec scolaire, et tout mène à penser que l'enseignement proposé par l'institution scolaire devrait être revu et devrait davantage intégrer la langue créole (Cellier, 1985 ; Lauret, 1991 ; Chaudenson, in Adelin et LebonEyquem, 2010), mais pas forcément pour des raisons de seuils linguistiques et de non prise en compte de la «langue maternelle »... Pour Adelin (2008), il a été pensé au niveau pédagogique des stratégies d'amélioration des compétences en français, partant d'un certain nombre de postulats (l'enfant a le créole comme langue maternelle, il convient de l'aider à atteindre un seuil de compétence minimal dans cette langue...), sans que les compétences de départ dans les deux langues de l'enfant n'aient été clairement établies. Si effectivement, l'enfant réunionnais possède des compétences en créole qui ne sont pas toujours prises en compte, son « déjà-là » en français ne doit pas non plus être nié. Si l'on adopte le point de vue de Cummins, dans laquelle de ses langues serait-il alors plus judicieux de faire atteindre en tout premier lieu un seuil linguistique?

\subsection{Les différents types de bilinguisme}

Un autre point d'achoppement théorique concerne la classification des formes de bilinguisme, classification découlant de la pensée dichotomique L1/L2. On a ainsi coutume de distinguer différentes formes de bilinguisme (Hamers et Blanc, 1983) :

le bilinguisme précoce (avant 4 ans), le bilinguisme successif (après 4 ans), et le bilinguisme tardif (après 6 ans : cas d'acquisition L2);

le bilinguisme équilibré et le bilinguisme dominant, eu égard au niveau de compétences linguistiques atteint dans chaque langue ;

le bilinguisme additif (bilinguisme "harmonieux », conduisant à des avantages cognitifs) et le bilinguisme soustractif (langues en relation de compétition, l'une menaçant l'autre, au risque d'entraîner des désavantages cognitifs et identitaires).

Dans le cadre théorique du macrosystème interlectal, cette façon de définir le bilinguisme se révèle non pertinente : de la même manière qu'il est inopérant de définir la langue maternelle de l'enfant réunionnais, il est illusoire de vouloir définir le caractère précoce ou tardif du bilinguisme. Tous les enfants réunionnais seraient à considérer comme des bilingues précoces, tandis que les terminologies de bilingue successif / tardif pourraient s'appliquer aux primo-arrivants non réunionnais.

Les notions de bilinguisme équilibré ou dominant s'annihilent également selon l'hypothèse de la macrolangue. La qualification en termes de bilinguisme additif versus bilinguisme soustractif, qui fait référence à la théorie des seuils de Cummins (le bilinguisme est soustractif quand les seuils n'ont pas été atteints, il est additif lorsqu'ils sont atteints ou dépassés), est, pour les mêmes raisons que celles avancées précédemment, critiquable sur le plan théorique. Il n'empêche toutefois que l'on atteste à La Réunion des situations évoquant un bilinguisme soustractif (enfant peu performant, peu à l'aise, dans ses langues) : mais la raison est-elle liée à la question des seuils linguistiques, ou est-elle à rechercher ailleurs ? Le statut sociolinguistique des langues parlées par l'enfant, le positionnement de la famille, qui choisit de transmettre ou de ne pas transmettre tel code linguistique, les contextes socio-culturel et socioéconomique, sont autant de facteurs influençant le bilinguisme sans qu'il soit forcément plus pertinent de 
parler de bilinguisme soustractif ou additif. Il est davantage question ici d'un handicap social que d'un handicap linguistique.

\subsection{La trajectoire du développement langagier}

Raisonner en termes de macrosystème interlectal amène également à s'interroger sur ce qui est avancé concernant la trajectoire développementale des enfants bilingues : est-il plus pertinent de considérer que les jeunes enfants réunionnais sont des monolingues réunionnais, car possédant un macrosystème linguistique, ou alors faut-il les considérer comme des sujets bilingues et penser leur acquisition langagière de la sorte ? Ainsi, observe-t-on chez les enfants réunionnais des phénomènes témoignant d'une séparation du français et du créole, ou d'une interdépendance, tels que des accélérations lors de l'acquisition, des phénomènes de décélération, de transfert de structures linguistiques?

Certains travaux réalisés dans une optique professionnelle (recherches menées par des orthophonistes exerçant à La Réunion : Mercier et ELO-SORR, 2012 ; Noël, 2012 ; Pichori, 2013) mettent en évidence des différences fondamentales entre les enfants monolingues francophones et les enfants réunionnais. Ainsi, les compétences en français des jeunes enfants réunionnais sont significativement plus faibles que celles des enfants métropolitains, ce qui plaide en faveur de différences sur le plan de l'acquisition. Le profil des enfants réunionnais s'apparenterait bien à celui d'enfants bilingues, chez qui il est objectivé un délai dans les acquisitions linguistiques. Cependant, ces études réalisées n'approfondissent pas les analyses linguistiques, et ne permettent pas d'argumenter l'hypothèse d'un rythme acquisitionnel marqué, par exemple, par des phases d'accélération ou de décélération, comme chez d'autres enfants bilingues. De nouvelles études seraient souhaitables, comparant par exemple le langage des enfants réunionnais à celui d'autres populations bilingues, ou se focalisant sur les étapes du développement langagier des jeunes enfants réunionnais. Un nouveau challenge s'offre à la recherche réunionnaise.

Si la théorie du macrosystème nous amène à remettre en question tant de termes et de notions, et nous amène, finalement, à remettre même en question le terme de bilinguisme pour parler des enfants réunionnais, il convient alors d'imaginer d'imaginer de nouveaux concepts, complétant ou se substituant à ceux que nous venons d'interroger. Ces concepts nous fournissent un nouveau cadre à penser l'acquisition langagière des jeunes enfants réunionnais.

\section{De nouveaux cadres à penser}

\subsection{De la L1 au lecte dominant}

Si le raisonnement en termes de L1 / L2 apparaît inadéquat en situation linguistique réunionnaise, il reste néanmoins envisageable, et même souhaitable pour l'avancée de la recherche dans le domaine de la psycholinguistique réunionnaise, de chercher à proposer un nouveau cadre théorique. Celui-ci consisterait en un ensemble de réajustements théoriques.

Pour Simonin (2002), s'il n'est pas souhaitable de parler de langue maternelle, on pourrait alors parler de langue dominante et de langue mineure. L'idée est intéressante mais doit être nuancée à plusieurs niveaux. Tout d'abord, notons que l'emploi de la terminologie «langue dominante» versus «langue mineure » renvoie à la pensée diglossique, alors qu'il n'est pas ici question de statut sociolinguistique mais de compétence linguistique. Il conviendrait ensuite non pas de parler de langue, mais de lecte. On peut enfin se demander si les jeunes enfants réunionnais ont forcément un lecte dominant et un lecte mineur : n'est-il pas plausible, de la même manière qu'il existe des cas de bilinguisme dominant et des cas de bilinguisme équilibré, d'envisager dans certains cas que les lectes soient équilibrés, équi-dominants ? Il apparaît effectivement judicieux de raisonner en termes de dominance de lecte, en admettant qu'il puisse ne pas y avoir de dominance. Pour ce faire, il convient de cerner le contexte linguistique de l'enfant, en s'appuyant sur une liste de facteurs favorisants, inspirés des travaux de Paradis (2007b) et de Lebon-Eyquem (2007) : 


\begin{tabular}{|l|l|}
\hline Conditions socio-psychologiques & $\begin{array}{l}\text { Valorisation des langues } \\
\text { Milieu socio-économique favorisant } \\
\text { Motivation à l'acquisition des lectes }\end{array}$ \\
\hline Aptitudes personnelles & $\begin{array}{l}\text { Capacités cognitives de l'enfant } \\
\text { Position sociale de meneur } \\
\text { Personnalité extravertie }\end{array}$ \\
\hline $\begin{array}{l}\text { Âge d'acquisition et fréquence d'exposition aux } \\
\text { différents lectes }\end{array}$ & $\begin{array}{l}\text { Quels locuteurs et quels lectes } \\
\text { Depuis quel âge } \\
\text { En gardant à l'esprit que français et créole sont, } \\
\text { depuis le jeune âge, présents dans l'environnement } \\
\text { de l'enfant }\end{array}$ \\
\hline Connaissance des caractéristiques des langues & $\begin{array}{l}\text { Conscientisation de la présence de différents lectes } \\
\text { Similitudes } \\
\text { Dissemblances }\end{array}$ \\
\hline Contextes d'input linguistique & $\begin{array}{l}\text { Dans tous les contextes : attention conjointe, } \\
\text { fréquence et qualité des inputs, apprentissage des } \\
\text { règles pragmatiques et des règles d'usage, } \\
\text { stimulation des capacités d'abstraction }\end{array}$ \\
\hline $\begin{array}{l}\text { Dans le milieu scolaire: association d'un lecte à } \\
\text { une situation, absence de pression normative, prise } \\
\text { en compte des approximations langagières }\end{array}$ \\
\hline $\begin{array}{l}\text { Dans le milieu familial : place dans la fratrie, } \\
\text { association d'un lecte à une situation, pratique de la } \\
\text { lecture }\end{array}$ \\
\hline $\begin{array}{l}\text { Dans la sphère des loisirs : association d'un lecte à } \\
\text { un tour de parole }\end{array}$ \\
\hline
\end{tabular}

Tableau 2. Facteurs favorisant et permettant l'établissement du lecte dominant

Cette liste permet, au vu des informations recueillies, de déterminer s'il existe ou non un lecte dominant ; elle permet également de juger si les conditions sont favorables à une acquisition langagière sans heurts (un «bilinguisme épanoui ») et, le cas échéant, permet d'apporter des pistes pour favoriser le développement du langage.

Si nous avons longuement discuté de l'acquisition langagière de manière générale, il convient de préciser que chez les monolingues comme chez les bilingues, les différences interindividuelles sont nombreuses. Le tableau 2 nous offre justement un aperçu des multiples facteurs pouvant influer sur l'acquisition langagière. Aussi il n'y a pas une seule manière d'être un locuteur réunionnais, et c'est ce que nous proposons d'aborder dans le point suivant.

\subsection{Comment est-on bilingue à La Réunion ? Les profils linguistiques}

S'il convient de ne plus parler désormais de L1 et de L2, on ne peut pour autant se contenter de dire que l'enfant réunionnais est plurilingue (ou devrait-on plutôt dire, plurilecte). Nous avons pu constater au début de notre développement le caractère panaché des pratiques langagières des jeunes enfants réunionnais; si l'intérêt de pouvoir identifier et qualifier les différentes productions des enfants est indéniable (ce que nous permet la classification de Lebon-Eyquem, 2010b), se pose la question des enfants qui les produisent. Observe-t-on chez tous les enfants réunionnais ces pratiques langagières ? Ou certaines productions sont-elles constatées majoritairement chez certains enfants? Est-il alors possible 
d'établir des profils linguistiques ? Adelin (2008) est la première à raisonner en termes de profils linguistiques. Elle propose de distinguer :

- les enfants dominants en français ou en créole: ils n'utilisent qu'un seul lecte dans la plupart des situations, et produisent de nombreux énoncés interlectaux quand ils ne possèdent pas dans leur langue les structures linguistiques nécessaires. Ils comprennent les règles d'usage, mais ont du mal à les respecter lorsqu'il s'agit d'utiliser le code non habituel ;

et les enfants équidominants (sans dominance) :

- les bi-lingues: ils utilisent les codes en fonction des situations d'interaction, réalisent peu de mélanges et maîtrisent les règles d'usage ;

- les mix-lingues: ils n'utilisent jamais un code seul, mais produisent au contraire des énoncés interlectaux quelle que soit la situation de communication. Ils ne connaissent pas les règles d'usage.

Cependant cette chercheure laisse penser que le mélange s'apparente en réalité à une non maîtrise des codes, alors que le mélange peut au contraire être la marque d'une bonne compétence langagière et communicationnelle. D'autres recherches ont permis d'affiner cette description et d'aboutir à une classification plus proche de la réalité linguistique et du cadre théorique du macrosystème, qui figure dans le tableau ci-dessous (Noël, 2012) :

\begin{tabular}{|c|c|c|c|}
\hline \multirow{2}{*}{\multicolumn{2}{|c|}{ Les enfants dominants }} & \multicolumn{2}{|c|}{ Les enfants équidominants } \\
\hline & & Les bi-lingues & Les mix-lingues \\
\hline $\begin{array}{l}\text { En français : } \\
\text { - utilisent un seul lecte } \\
\text { dans la plupart des } \\
\text { situations } \\
\text { - productions } \\
\text { interlectales peu } \\
\text { nombreuses (le plus } \\
\text { souvent socialement } \\
\text { établies) } \\
\text { - comprennent et } \\
\text { appliquent les règles } \\
\text { d'usage }\end{array}$ & $\begin{array}{l}\text { En créole : } \\
\text { - utilisent un seul lecte } \\
\text { dans la plupart des } \\
\text { situations } \\
\text { - productions } \\
\text { interlectales peu } \\
\text { nombreuses (présentes } \\
\text { surtout quand le français } \\
\text { est ciblé) } \\
\text { - énoncés interlectaux } \\
\text { parfois non socialement } \\
\text { établis } \\
\text { - difficultés à respecter } \\
\text { les règles d'usage en } \\
\text { français }\end{array}$ & $\begin{array}{l}\text { - maîtrisent les règles } \\
\text { d'usage des deux langues } \\
\text { et celles des productions } \\
\text { interlectales } \\
\text { - énoncés interlectaux le } \\
\text { plus souvent socialement } \\
\text { établis }\end{array}$ & $\begin{array}{l}\text { - utilisation rare d'un } \\
\text { code linguistique seul } \\
\text { - production d'énoncés } \\
\text { interlectaux en tous } \\
\text { contextes } \\
\text { communicationnels } \\
\text { - énoncés interlectaux } \\
\text { souvent provisoires } \\
\text { - ne connaissent pas les } \\
\text { règles d'usage } \\
\text { - distinguent } \\
\text { difficilement le créole du } \\
\text { français }\end{array}$ \\
\hline
\end{tabular}

Tableau 3. Tableau des profils linguistiques révisés (Noël, 2012)

On constate que dans cette dernière classification, le mélange est attesté dans tous les profils, et n'est pas forcément révélateur de confusion ou autres difficultés langagières. On observe des différences de fréquence de production du mélange (aussi les enfants dominants en français produiraient moins de productions mélangeantes, tandis que les enfants équidominants en produiraient davantage), mais jamais de réelle absence de mélange ; le mélange est, selon les profils, plus ou moins socialement établi.

Raisonner en termes de profils linguistiques permet une catégorisation que l'on sait nécessaire, même lorsqu'elle s'avère complexe comme c'est le cas en situation de contact de langues; cette manière de procéder permet de donner sens aux productions linguistiques des jeunes enfants réunionnais, et montre que le mélange, s'il doit nécessairement être vu comme une caractéristique fondamentale du langage des Réunionnais, n'échappe pas à la description et n'a pas toujours la même fonction et la même signification. 
Penchons-nous enfin, avant de conclure, sur la question de l'organisation cognitive. Il n'existe pas à l'heure actuelle de consensus concernant la manière dont pourraient être organisés les systèmes linguistiques des sujets bilingues. Au vu du développement que nous venons d'effectuer, et des nouveaux concepts que nous venons de poser, que peut-on supposer du fonctionnement cognitif des locuteurs réunionnais? Quel éclairage peut, dans ce domaine, nous apporter la théorie du macrosystème interlectal ?

\subsection{L'organisation cognitive des sujets réunionnais}

Si le concept du macrosystème plaide pour l'existence d'un seul système langagier, d'une macrolangue, on peut alors postuler une organisation en un système linguistique unique, qui le resterait tout au long de la vie, ce que certains auteurs supposent concernant les locuteurs bilingues tardifs (Alario, 2012). Cette hypothèse est d'autant plus cohérente que français et créole réunionnais ont en commun plus de la moitié $\mathrm{du}$ système phonologique et du système lexical (Adelin et Lebon-Eyquem, 2010). Ceci soulève néanmoins quelques questions, qui peuvent apparaître comme des contre-arguments à l'hypothèse d'un système linguistique unique :

On atteste chez certains locuteurs réunionnais adultes un accent créole, et non chez d'autres. Pourquoi celui-ci persiste-t-il dans certaines situations, et quel sens lui donner en ce qui concerne la représentation phonologique? Pourquoi certains Réunionnais réagiraient-ils comme des « bilingues tardifs », selon l'hypothèse d'Alario (2012), alors que d'autres auraient plutôt un profil de « bilingues précoces » (sans accent marqué) ?

Sur le plan lexical, on peut observer chez certains locuteurs réunionnais des phénomènes évoquant la concurrence lexicale (le sujet souhaite donner un mot dans une des langues mais ne parvient pas à accéder au terme, et donne «à défaut » l'équivalent dans l'autre langue). Ces phénomènes sont alors similaires à ce que l'on peut attester dans des situations plurilingues où les langues sont relativement distinctes. Peut-on alors penser que le système lexical serait distinct pour le français et le créole, ce qui va à l'encontre de la théorie du macrosystème ? Dans ce cas, pourquoi alors ce phénomène ne s'atteste-t-il pas chez tous les locuteurs réunionnais?

- Les ressemblances morphosyntaxiques sont nombreuses entre le créole réunionnais et le français, mais les différences sont également importantes : comment tout ceci pourrait-il se combiner en un seul système linguistique?

Une récente recherche sur la fréquence lexicale à La Réunion (Lebon-Eyquem et Noël, à paraître) a montré qu'il existait des différences de fréquence entre le lexique métropolitain et le lexique réunionnais, mais que les sujets ne semblaient pas pour autant fonctionner en opposant langue française et langue créole. Aussi des contraintes de langue (donner plus de mots en créole ou plus de mots en français, utiliser lors du protocole une ou l'autre langue) n'avaient pas eu d'impact sur la nature des mots fournis : dans tous les contextes, les sujets interrogés avaient fourni des mots créoles et des mots français, indépendamment de la langue demandée ou de la langue parlée par l'examinateur, ce qui appuie l'hypothèse que les Réunionnais posséderaient un système lexical réunionnais unique, et non deux systèmes distincts (un pour chaque langue).

Néanmoins, le macrosystème interlectal, s'il amène à penser un système linguistique unique, ne nie pas l'existence de lectes distincts, sous-composantes de la macrolangue réunionnaise. Il est alors plausible que les représentations linguistiques soient uniques mais soient dans une certaine mesure souscompartimentées en fonction des lectes parlés, ce qui expliquerait l'absence ou la présence d'un accent, ou encore le phénomène de concurrence lexicale. 


\section{Conclusion}

Dans cet article, nous avons souhaité questionner un ensemble de concepts théoriques concernant le bilinguisme et l'acquisition langagière. Partant d'une situation linguistique particulière, la situation plurilingue de La Réunion, et adoptant l'éclairage offert par le concept du macrosystème interlectal, nous avons abordé différents points théoriques, comme la question de la langue maternelle, des seuils de compétence, de la typologie du bilinguisme, ou encore de l'organisation cognitive des langues des individus bilingues. Déjà contestés pour d'autres situations de bilinguisme, la notion de langue maternelle et les différents postulats théoriques qui en découlent se révèlent particulièrement inopérants en contexte réunionnais : peut-on parler de langue maternelle pour des enfants évoluant dans un milieu linguistique panaché, exposés dès leur jeune âge à un mélange à géométrie variable ? Peut-on, d'ailleurs, parler de bilinguisme, dans son sens premier, c'est-à-dire l'état d'individus ayant accès à plus d'un code linguistique ? Ne pourrions-nous pas considérer au contraire que la langue des enfants réunionnais est unique, est un "réunionnais », une macrolangue, ou, pour reprendre la proposition d'Abdelilah-Bauer (2008), est le bilinguisme?

De nombreuses questions, comme celle du schéma de l'acquisition, ou encore celle de l'organisation cognitive, restent ouvertes : de nouvelles études sur la situation linguistique réunionnaise, qui se révèle être une situation linguistique passionnante pour la recherche du fait de sa singularité, pourraient apporter des éléments de réponses intéressants et alimenteraient le débat portant sur l'unicité ou la scission du système linguistique des individus bilingues ou plurilingues.

Si nous avons au fil de cet article remis en question un certain nombre de notions, nous avons également montré qu'un nouveau cadre de pensée est envisageable, et semble davantage convenir aux situations linguistiques telles que celle de La Réunion : à la notion de langue maternelle peut se substituer la notion de lecte potentiellement dominant, à la place de la typologie classique des profils de bilinguisme peut se concevoir une typologie en termes de profils linguistiques. Le mélange, au premier abord insaisissable et indescriptible, peut finalement être analysé, même s'il reste par essence imprédictible et toujours novateur. Il faut pour cela se parer de nouveaux outils linguistiques, comme ceux proposés par Lebon-Eyquem (2010b) pour l'étude des productions langagières enfantines, mais il nous faut avant tout dépasser la vision réductrice du mélange-confusion. Ceci revient peut-être, alors, à changer de perspective : accepter l'absence d'unicité, le complexe, l'hétérogène, l'imprévisible, le non-monolingue, en somme penser le langage à travers le regard du bilingue, qui ne perçoit peut-être pas chez lui cette pluralité qu'on lui trouve.

Notre article se veut être l'état des lieux d'une démarche réflexive, et n'a pas ni la prétention d'être exhaustif dans son argumentation, ni celle d'être affirmatif dans ses conclusions. Il donne à lire une nouvelle manière d'appréhender certaines situations de bilinguisme, de concevoir le fonctionnement bilingue, et pourrait donner de nouvelles orientations à la recherche linguistique réunionnaise.

\section{Références bibliographiques}

Abdelilah-Bauer, B. (2008). Le défi des enfants bilingues. Grandir et vivre en parlant plusieurs langues. Paris : La Découverte.

Adelin, E. (2008). Créole et français de petits écoliers réunionnais. Prolégomènes à l'évaluation de langues proches. Thèse de doctorat. Université de La Réunion.

Adelin, E., et Lebon-Eyquem, M. (2010). Adaptation de la didactique du français aux situations de créolophonie. Guide du maître : La Réunion. Niveau : Cycle 2 (CP et CE1). Paris : OIF / LeWebPédagogique.

Alario, F.-X. (2012). Choisir des mots et les prononcer : les affres du bilinguisme ? In Gatignol, P., et Topouzkhanian, S. (Eds.), Bilinguisme et biculture : Nouveaux défis ?, Isbergues : Ortho Edition, 9-17.

Beniamino, M., et Baggioni, D. (1993). Le français, langue réunionnaise. In Robillard, D. (de), et Beniamino, M. (Eds.), Le français dans l'espace francophone, Paris : Champion, 151-172. 
Bhatia, T.-K., et Ritchie, W.C. (1999). The bilingual child: some issues and perspectives. In Bhatia, T.-K., et Ritchie, W.C. (Eds.), Handbook of child language acquisition, San Diego : Academic Press, 569-643.

Borel, S. (2004). Parler Biennois : Une approche holistique du bilinguisme à Biel-Bienne. Mémoire de Licence de Linguistique, sous la direction du Pr. GAJO L. Université de Neuchâtel.

Cellier, P. (1985). Comparaison syntaxique du créole réunionnais et du français (Réflexions pré-pédagogiques). Saint-Denis/Paris : Université de La Réunion/CNRS.

Chaudenson, R. (1993). Francophonie, français zéro, et français régional. In Robillard, D. (de), et Beniamino, M. (Eds.), Le français dans l'espace francophone, Paris : Champion, 385-405.

Coste, D., Moore, D., et Zarate, G. (1999). Compétence plurilingue et pluriculturelle. Strasbourg : Ed. du Conseil de l'Europe.

Crémieux, S. (2000). Aspects des politiques linguistiques familiales : comment le créole et le français sont-ils perçus et gérés par les parents réunionnais? Mémoire de maîtrise de Lettres Modernes, sous la direction de BAVOUX C. Université de La Réunion.

Cummins, J. (1976). The Influence of Bilingualism on Cognitive Growth: a Synthesis of Research Findings and Explanatory Hypothesis. Working Papers of Bilingualism, 9, 1-43.

Deprez, C. (1989). Le plurilinguisme des enfants à Paris. Revue européenne de migrations internationales, 5(2), 71-87.

Deprez, C. (2002). Le jeu des langues dans les familles bilingues d'origine étrangère. Estudios de Sociolingüística, 1, $59-74$.

Fioux, P. (2007). Bilinguisme et diglossie à l'île de la Réunion : contribution à l'histoire d'un débat sociolinguistique, 1974-2006. Paris : L'Harmattan.

Genelot, S., Negro, I., et Peslages, D. (2006). Compétences bilingues français / créole chez des enfants de 5 ans en contexte martiniquais. Etudes créoles, 28(2), 41-66.

Georger, F. (2005). Approche du système de communication linguistique de certains enfants réunionnais : réflexion sur le langage en situation de contact de langues. Mémoire de DEA Langage et Parole, sous la direction de PRUDENT L.-F. Université de La Réunion.

Golberg, H., Paradis, J., et Crago, M. (2008). Lexical acquisition over time in minority first language children learning English as a second language. Applied Psycholinguistics, 29(1), 41-65.

Goldstein, B. A., et Fabiano, L. (2007). Assessment and Intervention for Bilingual Children with Phonological Disorders. The ASHA Leader, 12(2), 6-?

Hamers, J. F. (1997). Contact des langues. In Moreau, M.-L. (éd.), Sociolinguistique : les concepts de base, Liège : Mardaga, 94-100.

Hamers, J. F., et Blanc, M. (1983). Bilingualité et bilinguisme. Bruxelles : Mardaga.

Keshavarz, M.H., et Ingram, D. (2002). The early phonological development of a Farsi English bilingual child. International Journal of Bilingualism, 6, 255-269.

Köpke, B. (2007). Language attrition at the crossroads of brain, mind, and society. In Köpke, B., Schmid, M. S., Keijzer, M., et Dostert, S. (Eds.), Language attrition : Theoretical perspectives, Amsterdam : John Benjamins PC, 9-32.

Lauret, D. (1991). Le créole de la réussite. Saint-Denis : Ed. du Tramail.

Lavaur, J.-M. (1996). L'accès au lexique et la compréhension de textes chez le lecteur bilingue. In Hamon, J.-F. (éd.), Acquisition de la lecture et accès au sens de l'écrit : perspectives psychocognitives et psycholinguistiques, SaintDenis : Editions Faculté des Lettres et Sciences Humaines Université de La Réunion, 109-120.

Lebon-Eyquem, M. (2007). Une approche du développement langagier de l'enfant réunionnais dans la dynamique créole-français. Thèse de doctorat. Université de La Réunion.

Lebon-Eyquem, M. (2010a). Productions interlectales réunionnaises dans la dynamique créole-français. In Blanchet, P., et Martinez, P. (Eds.), Pratiques innovantes du plurilinguisme: émergence et prise en compte en situations francophones, Paris : Editions des archives contemporaines, 83-96. 
Lebon-Eyquem, M. (2010b). Evolution et impact de l'école et de l'environnement social sur les usages de 4 enfants de 3 ans en milieu créole. CMLF 2010, 1877-1895.

Lebon-Eyquem, M., et Noël, A. (à paraître). Etude de 21 catégories sémantiques et du phénomène de prototypie lexicale. Quelles normes catégorielles à La Réunion? Etudes créoles, ?- ?.

Ledegen, G. (2003). Regards sur l'évolution des mélanges codiques à La Réunion : l'avènement de l'interlecte ? In Ledegen, G. (éd.), Anciens et Nouveaux Plurilinguismes : Actes de la 6e Table Ronde du Moufia, Cortil-Wodon : E.M.E., 175-186.

Ledegen, G. (2012). Prédicats "flottants" entre le créole acrolectal et le français à La Réunion : exploration d'une zone ambiguë. In Chamoreau, C., et Goury, L. (Eds.), Systèmes prédicatifs des langues en contact, Paris : CNRS Editions, 251-270.

Lefebvre, F. (2008). Orthophonie et bilinguisme : élaboration d'un livret d'information à l'usage des orthophonistes. Mémoire d'orthophonie, sous la direction de CLOUARD C. Université de Nantes.

Lindholm, K. (1980). Bilingual children: Some interpretations of cognitive and linguistic development. Children's Language, 215-266.

Mackey, W. F. (1997). Bilinguisme. In Moreau, M.-L. (éd.), Sociolinguistique: les concepts de base, Liège : Mardaga, 61-64.

Marvilliers, S. (2009). Les pratiques langagières d'un enfant d'origine mahoraise dans la société multilingue de La Réunion : effets des normes? Actes du colloque AcquisiLyon 09, 1-4.

Mercier, S., et ELO-SORR (2012). Evaluation clinique standardisée dans un milieu diglotte : la problématique réunionnaise et l'étalonnage réunionnais de la batterie d'Evaluation du Langage Oral de A. Khomsi. In Gatignol, P., et Topouzkhanian, S. (Eds.), Bilinguisme et biculture : Nouveaux défis ?, Isbergues : Ortho Edition, 203-229.

Monteil, C. (2010). Le créole encore très largement majoritaire. Economie de La Réunion: Revue de l'INSEE, 137, 4-6.

Moore, D. (2006). Plurilinguismes et école. Paris : Didier.

Noël, A. (2012). Intérêt de l'analyse des représentations linguistiques et des pratiques langagières à La Réunion dans la pratique orthophonique. Pour une meilleure évaluation des enfants réunionnais. Mémoire de master en Sciences du Langage, sous la direction de LEBON-EYQUEM M. Université de La Réunion.

Paradis, J. (2001). Do bilingual two-year-olds have separate phonological systems? The International Journal of Bilingual Education and Bilingualism, 5(1), 19-38.

Paradis, J. (2007a). Bilingual Children with Specific Language Impairment: Theoretical and Applied Issues. Applied Psycholinguistics, 28(3), 551-564.

Paradis, J. (2007b). Second Language Acquisition in Childhood. In Hoff, E., et Shatz, M. (Eds.), Handbook of Language Development, Oxford : Blackwell Publishing Ltd, 387-406.

Paradis, J., Crago, M., et Bélanger, C. (2005a). Le développement langagier bilingue chez les enfants : incidence sur l'évaluation du trouble primaire du langage. Fréquences, 17(3), 27-30.

Paradis, J., Crago, M., et Genesee, F. (2005b). Domain-General versus Domain-Specific Accounts of Specific Language Impairment: Evidence from bilingual children's acquisition of Object Pronouns. Language Acquisition, 13(1), 33-62.

Paradis, J., Crago, M., Genesee, F., et Rice, M. (2003). French-English Bilingual Children with SLI : How do they compare with their Monolingual Peers? Journal of Speech, Language and Hearing Research, 46(1), 113-127.

Pichori, N. (2013). Pertinence d'un étalonnage spécifique de six épreuve de l'EVALO 2-6 chez l'enfant réunionnais de 4 ans 3 mois à 6 ans 3 mois. Mémoire d'orthophonie, sous la direction de COSSARD A. Université de Bordeaux.

Prudent, L.-F. (1981). Diglossie et interlecte. Langages, 15(61), 13-38.

Rapanoël, S., et Ledegen, G. (2007). Triade langagière d'enfants migrants à La Réunion. Diversité, 151, 161-169. 
Rivera Mindt, M., Arentoft, A., Kubo Germano, K., D’Aquila, E., Scheiner, D., Pizzirusso, M., Sandoval, T.C., et Gollan, T.H. (2008). Neuropsychological, Cognitive, and Theoretical Considerations for Evaluation of Bilingual Individuals. Neuropsychology Review, 18(3), 255-268.

Simonin, J. (2002). Parler réunionnais ? Hermès, 32-33, 287-296.

Souprayen-Cavery, L. (2010). L'interlecte réunionnais. Paris : L'Harmattan.

Tirvassen, R. (2003). Approcher les contacts des langues à partir des outils issus du structuralisme: quel usage effectuer du terme interférence? In Ledegen, G. (éd.), Anciens et Nouveaux Plurilinguismes: Actes de la 6e Table Ronde du Moufia, Cortil-Wodon : E.M.E., 211-229.

Weinreich, U. (1953). Languages in Contact: Findings and Problems. New York : Linguistic Circle of New York. 